\title{
Digital Video Watermarking using DWT for Data Security
}

\author{
Chitrasen $^{1}$, Tanuja Kashyap ${ }^{2}$ \\ Designation, Department, College, City, Country M.Tech Research Scholar, Department of Electronics and \\ Telecommunication, Bhilai Institute of Technology, Durg, India ${ }^{1}$ \\ Associate Professor, Department of Electronics and Telecommunication, Bhilai Institute of Technology, Durg, India ${ }^{2}$

\begin{abstract}
The Problem of illegal manipulation and distribution of digital video is becoming a big issue. To solve this problem, a new technology has been proposed. Basically, it is a process of embedding copy right information into bit streams of any video. In this particular scheme, the randomly segmentation and reconstruction of embedded secrete data is done without knowing the original host video. During the embedding process, secret data is embedded in individual video frames using the DWT's frequency domains. In this proposed work, a DWT video watermark technique has been introduced for securing data. We proposed a Haar wavelet based digital watermark technique to secure data. In Haar wavelet, Dyadic equation is used to embed the copy right information in video bit streams.
\end{abstract}

Keywords: Digital video, DWT, Haar wavelet filter, Dyadic Equation

\section{INTRODUCTION}

Watermarking is a technique for securing various copyright data such as text, image, audio and video. Copyright protection is the major part of the watermarking. Since 13th century, Digital Watermarking came into existence [1]. In case visible watermarks, visual patterns like logos were used to get inserted into digital data. To convey the hidden information, most watermarking systems involve marking alteration on the cover data. This technique is known to be invisible watermarks. On the other hand, digital watermarks are found with the advancement of internet. Thus, the idea of watermarking into digital data can be naturally extended. Due to proliferation of digital data (image, audio, and video), popularity of digital watermarking has been increased. Recently the copy right of digital images and to verify the multimedia data integrity, numerous digital watermarking algorithms are developed [2], [3].

Mainly due to the superior energy compaction property of wavelets, the use of the wavelets in image and video coding has increased significantly over the years. The minimum requirement for a watermark is being invisible. Many authors require a watermark image to have the same quality as the original one; this has been found after studying several research papers. A number of properties of wavelet transform will be discussed in next section.

\section{Literature SURVEY}

Min-Jeong Lee et al. proposed a practical video watermarking technique on the compressed domain that is real-time and robust against video processing attacks. In particular, they focused on video processing that is commonly used in practice such as downscaling resolution, framenrate changing, and transcoding. [2]

Suppat Rungraungsilp et al. proposed QR Code (Quick Response Code) embedded technique for invisible watermarking by using Discrete-Fourier-Transform (DFT) compare with Discrete-Wavelet-Transform (DWT). [3]
Shanjun Zhang et al. proposed a novel watermarking method to embed QR codes in digital images. The method is based on discrete wavelet transform (DWT). [4]

G. Langelaar et al. proposed an innovative practical video watermarking scheme based on MPEG-2. Watermark can be extracted blindly without original images and complete decoding is unnecessary. [5]

Sanjana Sinha et al. proposed a hybrid digital video watermarking scheme based on Discrete Wavelet Transform (DWT) and Principal Component Analysis (PCA). [6]

S.Nafees Ahmed et al. proposed video data embedding scheme the embedded secret data is randomly segmented and reconstructed without knowing the original host video. Secret data is embedded in individual video frames using the frequency domains of DWT. [7]

\section{III.THE WAVELET Transform}

Mathematical Technique i.e. DWT is utilized in the algorithm watermarking. In a wide variety of signal processing application discrete wavelet transform is used. Decomposition of an image or a video frame into sub images is done by 3D DWT. The sub image resembles the original on $1 / 4$ the scale of original during approximation. Frequency Band of an image is separated into lower resolution approximation sub-band, horizontal, vertical, diagonal detail components robustness increases with respect to attacks by embedding the watermark in low frequencies.

High frequency sub-band is less sensitive to high frequencies; watermark becomes more imperceptible due to embedding of watermark in high frequency sub-bands. While it becomes more robust against variety of attacks such as filtering, lossy compression and geometric distortion, due to embedding in low frequencies. [6] 
International Journal of Advanced Research in Computer and Communication Engineering Vol. 4, Issue 1, January 2015

\begin{tabular}{|c|c|}
\hline LL1 & $H L_{1}$ \\
\hline LH 1 & $H H_{1}$ \\
\hline
\end{tabular}

Fig.1 DWT sub-bands

Basically, four coefficient images are divided using discrete wavelet transform in the single level. 3D DWT generates four coefficients: LL, LH, HL, and HH. Here $\mathrm{HH}$ is diagonal high frequency band, HL is vertical high frequency band, LH is horizontal high frequency band and LL represents low frequency band. In high altitudes, the most prominent information get appears likewise the less prominent information appears in very low altitudes. Data compression can be achieved by discarding these low altitudes. High compression ratio with good quality of reconstruction is enabled by wavelet transform. As compared to FFT or DCT, DWT is believed to the more accurate model of human visual system. [7]

\section{IV.METHODOLOGY}

In this proposed design, a random video has been taken as input. Sample frame of this input video is shown in Fig. 3. Now after applying Haar wavelet transform we are getting four parts i.e. LL, LH, HL and $\mathrm{HH}$ parts as shown in Figure 3,4,5,6 respectively. In this process firstly, Sample frames of input video is converted into gray video frames. In the process of transformation, embedding process is applied on LL part. In which embedded watermark CS as shown in Fig. 8 got embedded with key as shown in Fig. 9. A watermarked frame after key embedding is finally obtained due to DWT.

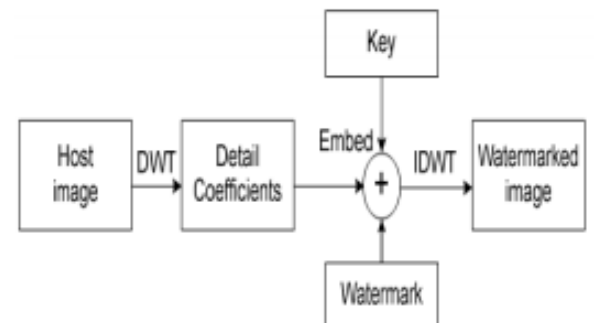

Fig. 2 Embedding Process

These obtained watermarked frames are converted back to watermarked video. After that recovery process is implemented. In this particular process, we again use to apply discrete wavelet transform to recover the watermarked image as shown in Fig. 11.

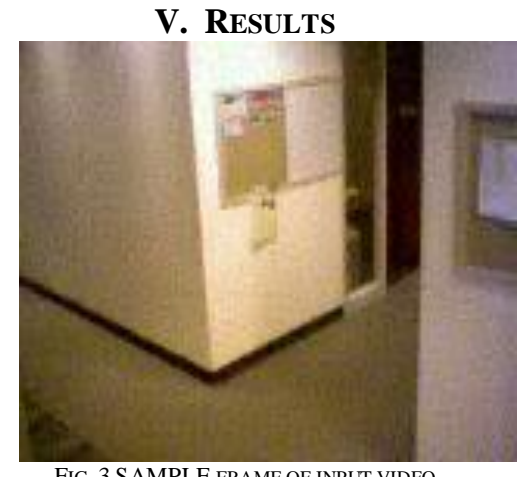

FIG. 3 SAMPLE FRAME OF INPUT VIDEO

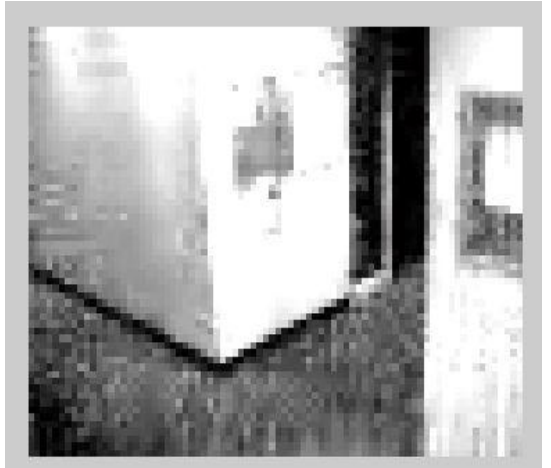

FIG.4 LL PART AFTER APPLYING HAAR WAVELET

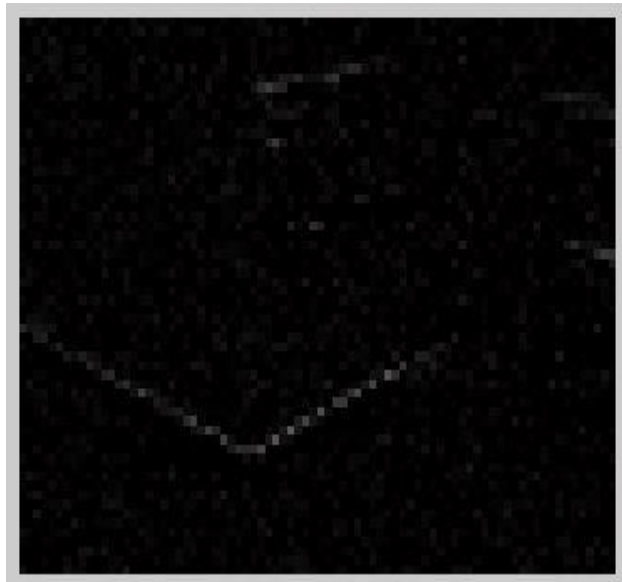

FIG. 5 LH PART AFTER APPLYING HAAR WAVELET

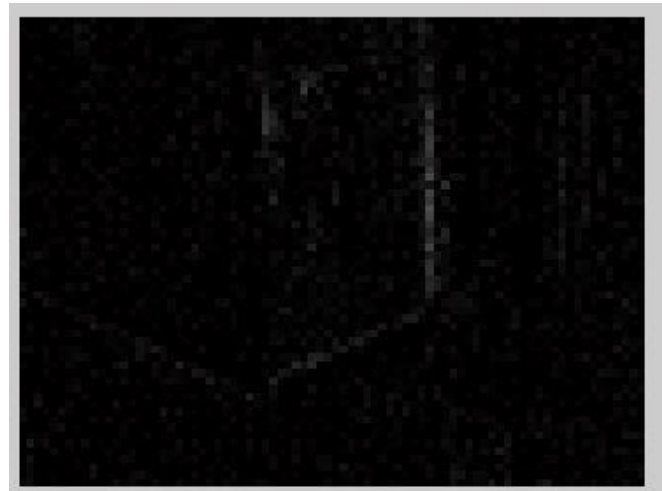

FIG.6 HL PART AFTER APPLYING HAAR WAVELET

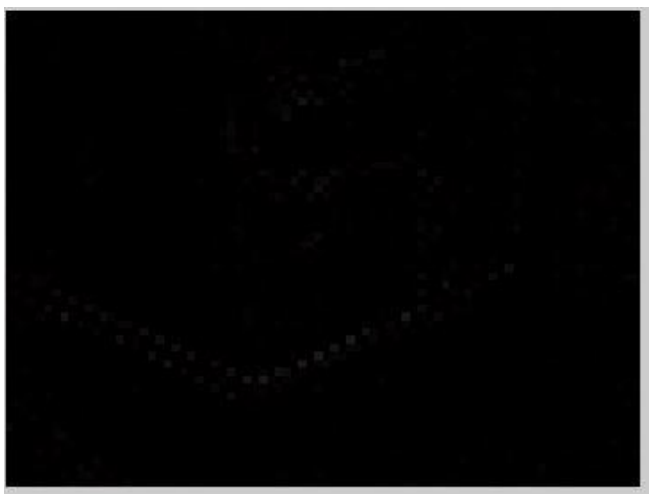

FIG. 7 HH PART AFTER APPLYING HAAR WAVELET 


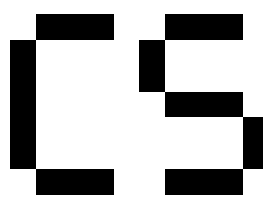

FIG.8 EMBEDDED KEY

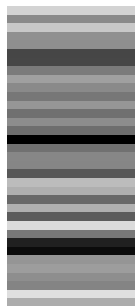

FIG.9 EMBEDDED KEY

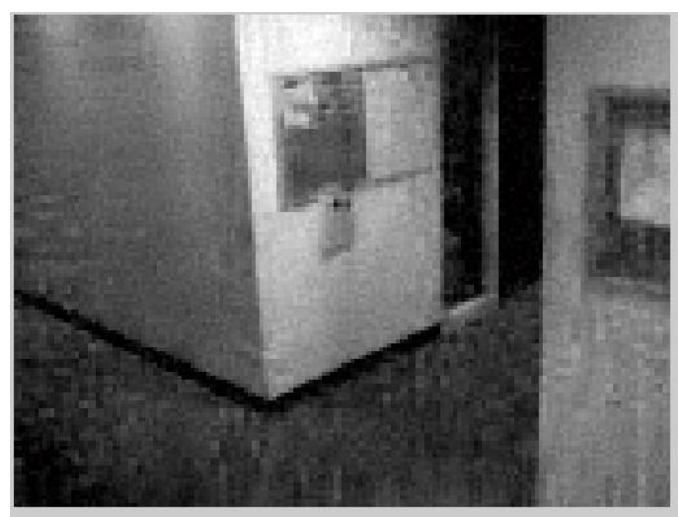

FIG. 10 WATERMARKED FRAME AFTER KEY EMBEDDING

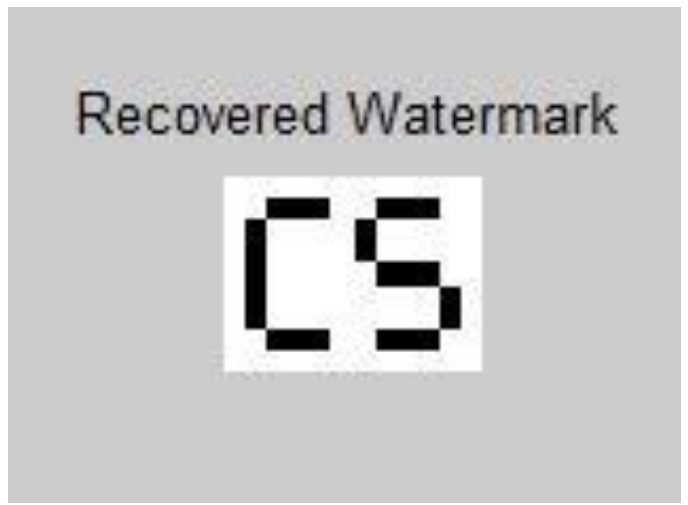

FIG. 11 RECOVERED WATERMARK IMAGE

\section{CONCLUSION AND FUTURE WORK}

Finally, a design has been implemented on Matlab that is satisfying the data security requirements using 2D DWT algorithm. In our proposed Design, We are using video watermarking technique for data hiding purpose. In this paper, Haar wavelet filter is used for watermark functionality against various geometrical distortions such as downscaling, cropping and rotation. In future for further improvement 3-D DWT will design.

\section{REFERENCES}

[1] Sadik. A.M .Al-Taweel, Putra Sumari, "Robust Video Watermarking Based On 3D-DWT Domain," IEEE Communications, 2009.

[2] Min-Jeong Lee, Dong-Hyuck Im, Hae-Yeoun Lee, Kyung-Su Kim, Heung-Kyu Lee, "Real-time video watermarking system on the compressed domain for high-definition video contents: Practical issues", Digital Signal Processing 22 (2012) 190-198.

[3] Suppat Rungraungsilp, Mahasak Ketcham, Tanee Wiputtikul, Kanchana Phonphak , and Sartid Vongpradhip," Data Hiding Method for QR Code Based on Watermark by comparing DFT with DWT Domain", International Conference on Computer and Communication Technologies (ICCCT'2012) May 26-27, 2012 Phuket.

[4] Shanjun Zhang, Kazuyoshi Yoshino," DWT-Based Watermarking Using QR Code", Science Journal of Kanagawa University 19 : 3-6 (2008).

[5] G. Langelaar, I. Setyawan, and R. Lagendijk, "Watermarking digital image and video data: A state-of-the-art overview," IEEE Signal Process. Mag., vol. 17, no. 5, pp. 20-46, Sep. 2000.

[6] Sanjana Sinha, Prajnat Bardhan, Swarnali Pramanick, Ankul Jagatramka, Dipak K. Kole, Aruna Chakraborty, "Digital Video Watermarking using Discrete Wavelet Transform and Principal Component Analysis" International Journal of Wisdom Based Computing, Vol. 1 (2), August 2011.

[7] S.Nafees Ahmed, B.Sridhar, Dr.C.Arun, "Robust Video Watermarking based on Discrete Wavelet Transform" International Journal of Computer Network and Security (IJCNS) Vol 4. No 1. Jan-Mar 2012.

[8] M.D. Swanson, M. Kobayashi, and A.H. Tewfik, Multimedia dataembedding and watermarking technologies, Proc. of the IEEE, vol. 86, no. 6, pp. $1064-1087$, Jun. 1998.

[9] I. Cox, J. Killian, T. Leighton, and T. Shamoon, "Secure spread spectrum watermarking for multimedia," IEEE Trans. Image Process., vol. 6, no.12, pp. 1673 -1687, Dec. 1997.

[10] F. Hartung and B. Girod, Digital Watermarking of MPEG-2 Coded Video in Bit stream Domain, Proc. IEEE International

[11] Conference on Acoustics, Speech and Signal Processing, ICASSP97, Vol.4, No.4, pp.2621-2624

[12] F. Hartung and B. Girod, "Watermarking of uncompressed and compressed video,” Signal Process., vol. 66, no. 5, pp. 283-301, May 1998.

[13] G. Langelaar and R. Lagendijk, Optimal differential energy watermarking of DCT encoded images and video[J], IEEE Transaction on Image Processing, vol. 10, no. 1, pp. 148-158, Jan. 2001

[14] Sun T F, Jiang X H, Shi S, et al. A novel differential energy video watermarking based on Watson visual model [C] International Symposium on Electronic Commerce and Security. Nanchang, China: Nanchang HangKong University, 2009: 179 - 183. 